\title{
A review of hippocampal activation in post-traumatic stress disorder
}

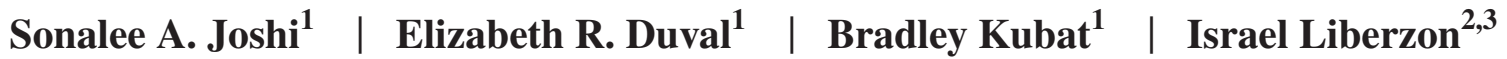

${ }^{1}$ Department of Psychiatry, University of Michigan Health System, Ann Arbor, Michigan

${ }^{2}$ Veterans Affairs Ann Arbor Healthcare System, Ann Arbor, Michigan

${ }^{3}$ Department of Psychiatry, Texas A\&M University Health Science Center, College Station, Texas

\section{Correspondence}

Sonalee A. Joshi, Department of Psychiatry, University of Michigan, 4250 Plymouth Rd., Ann Arbor, MI 48109.

Email: sojoshi@umich.edu

\begin{abstract}
Post-traumatic stress disorder (PTSD) is often characterized by deficits in memory encoding and retrieval and aberrant fear and extinction learning. The hippocampus plays a critical role in memory and contextual processing and has been implicated in intrinsic functional connectivity networks involved in self-referential thought and memory-related processes. This review focuses on hippocampal activation findings during memory and fear and extinction learning tasks, as well as resting state hippocampal connectivity in individuals with PTSD. A preponderance of functional neuroimaging studies to date, using memory, fear learning, and extinction tasks, report decreased or "controls comparable" hippocampal activation in individuals with PTSD, which is usually associated with poorer performance on the task imaged. Existing evidence thus raises the possibility that greater hippocampal recruitment in PTSD participants may be required for similar performance levels. Studies of resting state functional connectivity in PTSD predominantly report reduced within-network connectivity in the default mode network (DMN), as well as greater coupling between the DMN and salience network (SN) via the hippocampus. Together, these findings suggest that deficient hippocampal activation in PTSD may be associated with poorer performance during memory, extinction recall, and fear renewal tasks. Furthermore, studies of resting state connectivity implicate the hippocampus in decreased within-network DMN connectivity and greater coupling with SN regions characteristic of PTSD.
\end{abstract}

K E Y W O R D S

extinction learning, fear learning, fMRI, hippocampus, memory, PTSD, resting state

\section{1 | REVIEW OF HIPPOCAMPAL FUNCTION IN POST-TRAUMATIC STRESS DISORDER}

Post-traumatic stress disorder (PTSD) is a serious and debilitating condition with a lifetime prevalence of approximately $8 \%$ in the general population, with higher rates associated with exposure to multiple traumatic events (Kessler, 2000; Kessler et al., 2005; Tamburrino et al., 2015; Thompson, Gottesman, \& Zalewski, 2006). PTSD is characterized by recurrent traumatic memories as well as other trauma re-experiencing-dreams, flashbacks, etc. (American Psychiatric Association, 2013), as well as overgeneralization of fear responses and deficits in utilizing contextual information to disambiguate potential threat (Brown et al., 2013; Garfinkel et al., 2014; Levy-Gigi, Szabo, Richter-Levin, \& Kéri, 2014). Identifying neural mechanisms underlying PTSD and associated symptoms is important for the development of effective preventive and targeted treatment strategies. Recent studies examining neural underpinnings of PTSD symptoms are beginning to suggest patterns 
of increased activation in brain regions involved in emotion response and processing (e.g., amygdala, insula), decreased activation in regions involved in emotion regulation (e.g., prefrontal cortex, PFC), and altered or diminished activation in regions involved in memory modulation and contextualization (e.g., hippocampus and PFC; Duval, Javanbakht, \& Liberzon, 2015; Hayes, Vanelzakker, \& Shin, 2012; Shin \& Liberzon, 2010). Interestingly, while the consensus surrounding diminished activation during emotional regulation and extinction-related tasks in the medial and lateral PFC in PTSD has been emerging (Bremner et al., 1999; Rabinak et al., 2014), hippocampal function across relevant tasks in PTSD has been interrogated less often (with noted exceptions: Garfinkel et al., 2014; Jovanovic, Kazama, Bachevalier, \& Davis, 2012; Milad et al., 2009, 2007) or incorporated in theoretical formulations.

The hippocampus is implicated in emotional memory formation and contextualization (Dere, Pause, \& Pietrowsky, 2010), and a large body of the literature over the last 20 years has documented smaller hippocampal volumes in PTSD (Chao, Weiner, \& Neylan, 2013; Childress et al., 2013; Logue et al., 2018; for latest review, see also Nelson \& Tumpap 2017). Furthermore, evidence suggests that smaller hippocampal volume prior to trauma exposure is a risk factor for PTSD development and persistence (Gilbertson et al., 2002; Van Rooij et al., 2015). While original hypotheses regarding changes in hippocampal volumes in PTSD were based on cytotoxic effects of hypercortisolism (Bremner, 2006; Sapolsky, 2000), more recent hypotheses involve alternative mechanisms such as suppression of neurogenesis in the hippocampus due to stress exposure (Niibori et al., 2012), which can negatively impact cognitive functions like memory, contextualization, and learning (McEwen, 1999). In fact, patients with PTSD have been shown to have general deficits in memory, learning, and contextualization (Levy-Gigi et al., 2012; Vasterling et al., 2002), lending support to the idea that hippocampal function underlies important cognitive and emotional processes relevant to PTSD.

Given the evidence for abnormalities in hippocampal structure in PTSD, as well as deficits in hippocampal-dependent functions like memory and learning, the hippocampus appears to be an important target for further investigation and possible intervention. However, there is no clear consensus nor systematic examination of functional differences in hippocampus associated with PTSD using in vivo functional neuroimaging approaches. The goal of this review is to summarize the literature to date, focused on hippocampal function in PTSD, in an effort to synchronize findings and identify areas of concordance, as well as areas in need of further investigation.

A literature search was performed in PubMed, PsycINFO, and Web of Science databases. Search terms were chosen based on previously studied functions of the hippocampus and behavioral deficits in PTSD (Eichenbaum, 2017; Janak \& Tye, 2015; McCormick, Rosenthal, Miller, \& Maguire, 2018;
Opitz, 2014; Sierra-Mercado, Padilla-Coreano, \& Quirk, 2011). As structural differences have been addressed more extensively in the literature (Chao, Weiner, \& Neylan, 2013; Childress et al., 2013; Logue et al., 2018; Nelson \& Tumpap 2017), this review focused solely on hippocampal function. Search terms included combinations of the following: [hippocampus or hippocampal activation or hippocampal function] and [PTSD or post-traumatic stress disorder], fMRI, [fear learning or fear conditioning], [fear generalization or memory], and [context, context processing], or resting state. The search was constrained to articles published in the last 10 years (since 2008). This search initially yielded 41 articles. Twelve manuscripts were then excluded from further review for not reporting empirical fMRI data with results assessing activation or resting state functional connectivity in the hippocampus, and 10 articles were excluded because they did not specifically examine participants with diagnosed PTSD. This resulted in 19 articles published from 2008 to 2017 utilizing a variety of tasks targeting hippocampal activation in PTSD participants. A meta-analysis was not conducted, as the goal of this review was to assess PTSD-related differences across a range of functions associated with the hippocampus, thus combining all the studies in a single meta-analysis would not be informative. The included studies can be grouped into three general categories: memory (semantic, episodic) tasks, fear-associated learning (acquisition, extinction, extinction recall, fear renewal) tasks, and resting state connectivity task. We have thus organized our findings accordingly.

\section{2 | MEMORY AND LEARNING}

\section{1 | Functional neuroimaging studies of memory in PTSD}

The key role that the hippocampus plays in a number of memory processes has been well established (for latest reviews, see Eichenbaum, 2017; Opitz, 2014). The hippocampus is critically involved in declarative memory (Cohen \& Squire, 1980; Eichenbaum \& Cohen, 2014; Huijgen \& Samson, 2015) encoding (Brown et al., 2014; Carrión, Haas, Garrett, Song, \& Reiss, 2010; Geuze, Vermetten, Ruf, de Kloet, \& Westenberg, 2008; Hayes et al., 2011), or the storage of new stimuli in memory retrieval (Cisler, Bush, James, Smitherman, \& Kilts, 2015), or recall of stored information, with research demonstrating that hippocampal damage elicits deficits in both semantic and episodic memory (Allen, 2018; Scoville \& Milner, 1957). Some models suggest that the hippocampus plays a primary role mainly in early encoding and retrieval (Bergstrom, 2016; Frankland \& Bontempi, 2005), with others purporting that the hippocampus is continuously involved in shaping memory retrieval over time (Bergstrom, 2016; Nadel \& Moscovitch, 1997). Whether hippocampus is critical solely to original encoding, or encoding 
and maintenance of memories, both the original encoding of traumatic memory and maintenance (expressed as intrusive symptoms) have been postulated as key mechanisms involved in PTSD development (Meyer et al., 2018). Hence, research on neural correlates of memory processes in the hippocampus is highly relevant to elucidation of PTSD pathophysiology.

Memory deficits have been well documented in PTSD (Brown et al., 2014; Carrión et al., 2010; Geuze et al., 2008; Hayes et al., 2011), with behavioral data suggesting that PTSD participants perform more poorly on memory tasks involving both neutral (Samuelson, 2011) and emotional (Brohawn, Offringa, Pfaff, Hughes, \& Shin, 2010; Ehlers \& Clark, 2000) information. PTSD participants had diminished semantic memory for neutral words compared to both asymptomatic trauma-exposed controls (TEC) and nontraumaexposed controls (NEC; Carrión et al., 2010; Geuze et al., 2008). Similarly, PTSD participants have demonstrated less detailed memory for episodic autobiographical and future imagined events compared to TEC (Brown et al., 2014). Together, these findings are quite consistent but rather counterintuitive, as theoretical conceptualizations of PTSD often postulate hyperactivity in the hippocampus in conjunction with memory performance. On the other hand, empirical evidence supporting hyperactivity in the hippocampus associated with general or trauma-linked memory processes in PTSD beyond subjective symptom reporting is still missing.

\subsection{Semantic memory encoding in PTSD}

A number of studies have examined semantic memory encoding and have reported hypoactivation or comparable activation to controls in the hippocampus in PTSD during memory encoding, which was associated with poorer performance during later retrieval. The majority of these studies identified the hippocampus as an a priori region of interest and examined activation using an AAL (automated anatomical labeling) mask; however, one study identified hippocampal activation through an exploratory whole brain analysis (Werner et al., 2009) and another conducted an ROI analysis using activation-based hippocampal coordinates (Geuze et al., 2008). One study reported decreased hippocampal activation in PTSD participants compared to TEC during encoding of negative images. Less hippocampal activation was associated with poorer retrieval of negative images, due to a greater false alarm rate in PTSD participants (Hayes et al., 2011). Two studies that reported levels of hippocampal activation in PTSD that were comparable to controls during encoding of neutral words also reported poorer performance on later retrieval tasks in PTSD participants (Carrión et al., 2010; Geuze et al., 2008). This suggests that similar levels of hippocampal activation between PTSD and control groups during encoding might not be sufficient to support similar performance between groups. One study using neutral words and another using negative emotional words reported greater hippocampal activation in PTSD participants during memory encoding. This greater hippocampal activation was, however, associated with memory performance in PTSD that was similar to that of the control participants (Thomaes et al., 2009; Werner et al., 2009). Together, these studies support the notion that greater hippocampal recruitment in PTSD during memory encoding is required for performance comparable to that seen in control participants. In addition, failure to activate hippocampus to a similar degree as healthy individuals during encoding was associated with poorer retrieval of encoded information in individuals with PTSD.

\subsection{Semantic memory retrieval in PTSD}

While altered hippocampal function during encoding may contribute to memory alteration in PTSD, it is possible that differences in hippocampal activation during memory retrieval are also related to PTSD-associated memory deficits. Five studies examined hippocampal activation during retrieval in individuals with PTSD compared to NEC. Similarly to research on memory encoding, studies of memory retrieval also primarily identified hippocampal function using an anatomical hippocampal mask, as the hippocampus was identified as an a priori region of interest. In addition, a few studies first conducted exploratory whole brain analyses to identify the hippocampus as a region of interest for subsequent analysis (Geuze et al., 2008; Hayes et al., 2011). Three of these studies (Carrión et al., 2010; Cisler et al., 2015; Geuze et al., 2008) reported lower hippocampal activation during retrieval of neutral words in PTSD, with no group differences during prior encoding. This lower activation during retrieval was associated with poorer semantic memory performance. Decreased hippocampal activation during memory retrieval has also been associated with greater symptomatology in individuals with PTSD (Carrión et al., 2010; Cisler et al., 2015). These findings suggest that reduced hippocampal activation in individuals with PTSD is associated with retrieval deficits and more severe symptoms.

In contrast, Thomaes et al. (2009) reported comparable hippocampal activation during memory retrieval and comparable performance for both negative and neutral words in PTSD and TEC participants. Hayes et al. (2011) did not report group differences in retrieval of neutral images in PTSD participants compared to NEC; however, decreased hippocampal activation was associated with decreased accuracy in retrieval of negative images in PTSD participants compared to NEC. Previous research has suggested differences in memory for emotional and nonemotional information (Sharot, \& Yonelinas, 2008), as evidence suggests that emotional memory is dependent on modulation of the hippocampus by the basolateral amygdala (BLA) and infralimbic mPFC (Pape \& Pare, 2010). These neural processes associated with retrieval 
of emotional compared to neural information may contribute to the decrease in hippocampal activation during retrieval of negative images for PTSD participants in this study.

\subsection{Episodic autobiographical retrieval in PTSD}

In addition to assessing hippocampal activation during semantic memory retrieval, some studies have examined hippocampal activation during retrieval of autobiographical and trauma-related narratives, using a whole brain analysis (Cisler et al., 2015) or an ROI analysis with the hippocampus identified as an a priori region of interest (St. Jacques, Botzung, Miles, \& Rubin, 2011). Although deficits in episodic memory retrieval in PTSD have been demonstrated (Brown et al., 2014), the role of hippocampal recruitment in this process remains unknown. Only one study has compared hippocampal activation in PTSD and NEC during retrieval of autobiographical memory. Compared to NEC, PTSD participants demonstrated increased hippocampal activation during retrieval of negative autobiographical memories and decreased hippocampal activation during retrieval of positive autobiographical memories (St. Jacques et al., 2011).

\section{3 | PAVLOVIAN FEAR LEARNING AND MEMORY IN POST- TRAUMATIC STRESS}

\subsection{Fear associative learning in PTSD}

Literature has also focused on the role of the hippocampus in modulating amygdala-dependent process of fear learning and memory in individuals with PTSD. Fear acquisition is primarily dependent on processing of fearful stimuli in the BLA moderated by activity in the midline and orbital PFC and the hippocampus (Janak \& Tye, 2015; Sierra-Mercado, Padilla-Coreano, \& Quirk, 2011). Although fear learning is associated with amygdala function (Janak \& Tye, 2015; Labar, Gatenby, Gore, Ledoux, \& Phelps, 1998), extinction learning, involving the processing of contextual information, has been associated with hippocampus and mPFC function (Liberzon \& Abelson, 2016, Figure 1; Maren, Phan, \& Liberzon, 2013). Therefore, in addition to probing the function of the amygdala, fear learning can be used to probe hippocampal-dependent contextual processing involved in fear modulation. Hippocampal-dependent contextual processing is critical in forming adaptive responses to ambiguous stimuli that signal fear or safety, depending on the context. Fear-associative learning paradigms (fear conditioning, extinction, extinction recall, fear renewal) allow one to test utilization of visual, cognitive, and instructional information comprising the context necessary for appropriate interpretation of ambiguous cues.

\section{2 | Fear acquisition}

To date, there is little evidence to suggest neurobiological or behavioral differences in fear acquisition in PTSD compared to controls (Garfinkel et al., 2014; Liberzon \& Abelson, 2016; Milad et al., 2009). PTSD participants do not differ from NEC or TEC in neural activation or skin conductance response (SCR) during fear acquisition in Pavlovian conditioning paradigms (Garfinkel et al., 2014, Milad et al., 2009; Steiger, Nees, Wicking, Lang, \& Flor, 2015). However, one study reported that greater hippocampal activation during fear acquisition in the PTSD group was associated with greater

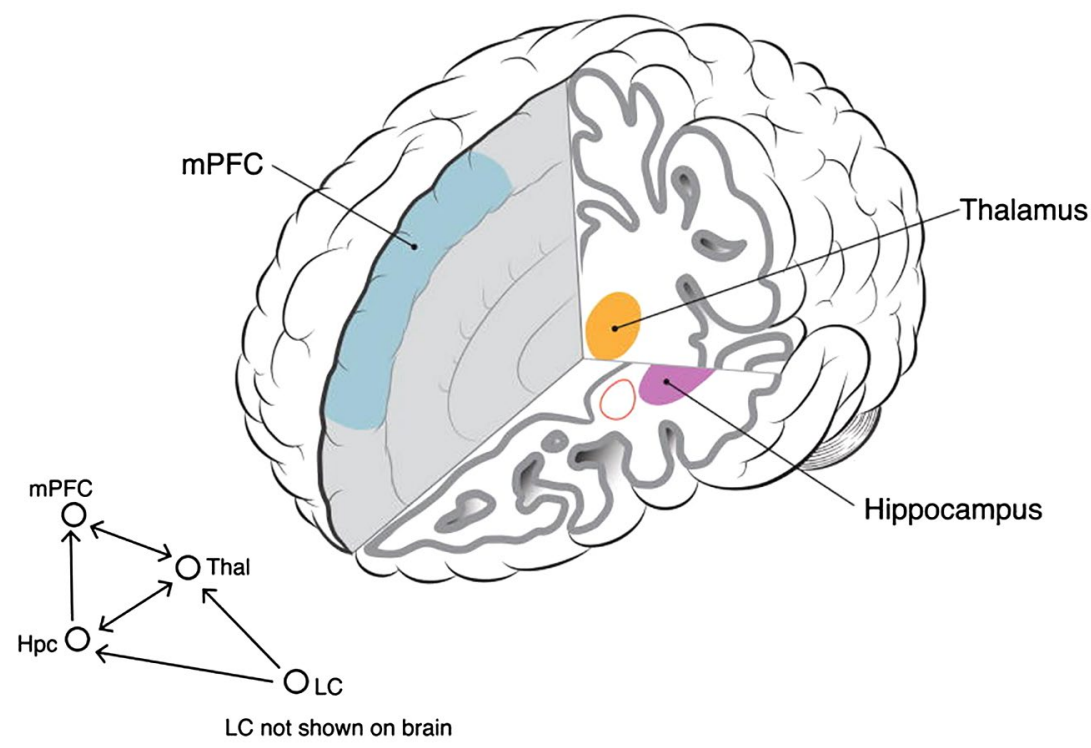

F I G U RE 1 Regions, including hippocampus, involved in fear extinction processes (from Liberzon \& Abelson, 2016) 
avoidance symptoms (Sripada, Garfinkel, \& Liberzon, 2013). These results raise the possibility that increased hippocampal activation might be present during fear acquisition in some individuals with more severe PTSD. Increased hippocampal activation was reported in PTSD in one study; however, this was observed during acquisition of contextual information. During a context acquisition phase in a context conditioning paradigm, increased hippocampal activation for PTSD compared to TEC and NEC was observed during "dangerous" context trials (Steiger et al., 2015).

\section{3 | Extinction learning, extinction recall, and fear renewal}

It is important to note that, while cued fear conditioning alone is context independent, fear extinction, and thus extinction recall and fear renewal, are dependent on contextual information and therefore likely require intact hippocampal function. Indeed, activation in the ventral hippocampus has been linked to contextual processing during extinction learning paradigms (Lopresto, Schipper, \& Homberg, 2016; Wotjak \& Pape, 2013). In concert, evidence suggests that hippocampaldependent context processing may be associated with extinction learning and recall in individuals with PTSD (Jovanovic et al., 2012; Liberzon \& Abelson, 2016). One study reported diminished SCR habituation during extinction recall in PTSD patients compared to TEC, which was associated with reduced hippocampal activation to extinguished conditioned stimulus (CS; Milad et al., 2009). However, another study failed to find group differences between PTSD and NEC or TEC during extinction recall (Steiger et al., 2015). The same group also reported increased hippocampal activation in PTSD compared to NEC during a modified ambiguous extinction recall/fear renewal phase; however, interpretation of these results is complicated as this was conducted in a novel context rather than following an $\mathrm{ABA}$ or $\mathrm{ABB}$ design used in other extinction learning studies (Wicking et al., 2016). Few studies have examined hippocampal activation during fear renewal in PTSD, but Garfinkel et al. (2014) reported lower hippocampal activation in PTSD compared to TEC participants and blunted SCR response during fear renewal.

Additional research should be conducted to understand how hippocampal activation underlying contextual processing relates to aberrant fear and extinction learning processes. Little evidence to date has supported group differences in hippocampal activation and behavioral performance during fear acquisition (Garfinkel et al., 2014; Liberzon \& Abelson, 2016; Milad et al., 2009; Steiger et al., 2015), and present findings suggest diminished or comparable to controls' hippocampal activation for PTSD during extinction recall and fear renewal (Garfinkel et al., 2014; Milad et al., 2009; Steiger et al., 2015). Prior research has shown that PTSD participants fail to appropriately recall extinction in a "safety" context or renew an appropriate fear response in a "threat" context (Garfinkel et al., 2014), indicating deficient utilization of contextual information. This inability to use contextual information to disambiguate potential safety and threat suggests that hippocampal-dependent contextual processing deficits might play an important role in altered fear responses in PTSD.

\section{4 | Resting state connectivity in PTSD}

While important information about the function of hippocampus and other regions is gained using specific task-based activation paradigms discussed above, additional information can be ascertained by examining intrinsic functional connectivity networks (ICNs) of the brain during rest. In the past decade, a large body of functional neuroimaging literature has documented the presence of ICNs, consisting of regions that demonstrate low frequency BOLD covariation with one another and are anticorrelated with BOLD variation in other regions, in the absence of a specific task (Fox, Snyder, Barch, Gusnard, \& Raichle, 2005). A number of resting state functional networks have been identified (Mitra \& Raichle, 2016; Power et al., 2011) and are related to personality traits (e.g., impulsiveness, trait anxiety), life experience, presence and duration of psychiatric symptoms, cognitive and motor abilities, and perception in healthy and disordered populations (Fornito \& Bullmore, 2010; Vaidya \& Gordon, 2013). Among the various ICNs, an important network of regions that are active at rest—default mode network (DMN) - has been identified and linked to mind wandering and self-directed thought (Greicius, Krasnow, Reiss, \& Menon, 2003). The hippocampus has been identified as a central node in the DMN, consistent with reports implicating the hippocampus in mind wandering, which often occurs during resting state assessment and involves episodic memory, visuospatial imagery, and mental time travel (McCormick et al., 2018). A number of studies have examined connectivity within the DMN and between the DMN and other networks, to investigate functional connectivity in PTSD using a seed-based whole brain analysis.

Overall, consistent findings of reduced resting state functional connectivity between regions within the DMN in PTSD participants compared to controls has been emerging (Patel, Spreng, Shin, \& Girard, 2012; Patriat, Birn, Keding, \& Harringa, 2016; Sripada et al., 2012). PTSD participants with (Miler et al., 2017) and without mild traumatic brain injuries (Patriat et al., 2016; Sripada et al., 2012) have exhibited reduced connectivity between the hippocampus and prefrontal regions, such as the mPFC, compared to TEC (Jin et al., 2014; Spielberg, McGlinchey, Milberg, \& Salat, 2015). This reduced within-DMN connectivity may contribute to characteristic symptoms of PTSD such as intrusive memories, dissociation, or avoidance (Akiki, Averill, \& Abdallah, 2017). 
PTSD participants with more severe avoidance and numbing symptoms had less functional connectivity within DMN, between the hippocampus and the posterior cingulate cortex (PCC), compared to TEC (Miler et al., 2017).

In healthy samples, BOLD signals in DMN regions are commonly inversely correlated with BOLD signals from regions of the $\mathrm{SN}$, which is typically active during decision making or attention-based tasks. Thus, healthy adults demonstrate segregation between DMN and SN. However, studies have demonstrated that participants with PTSD demonstrate less segregation between the DMN and $\mathrm{SN}$ regions, via the hippocampus (Akiki, Averill, \& Abdallah, 2017; Seeley et al., 2009; Sripada et al., 2012).

Overall, resting state findings suggest that PTSD participants may have less connectivity between the hippocampus and other regions of the DMN, compared to controls, and that the degree of within-DMN connectivity is associated with symptom severity. PTSD participants also demonstrate less anticorrelation between DMN and SN compared to controls, with these aberrant connections often involving the hippocampus.

\section{\begin{tabular}{l|l}
4 & DISCUSSION
\end{tabular}}

There is an increasing body of neuroimaging literature on hippocampal function in individuals with PTSD. We reviewed existing evidence for altered hippocampal activation during learning and memory task, and resting state functional connectivity in individuals with PTSD in the last decade. Overall, the preponderance of evidence suggests that decreased or comparable to controls' hippocampal activation in PTSD during encoding or retrieval of the memory trace, regardless of functional performance on memory and extinction retention tasks with these levels of hippocampal recruitment, is poorer. Overall, present evidence suggests that decreased or comparable activation in individuals with PTSD compared to controls during memory encoding and retrieval is associated with poorer performance on memory and extinction retention tasks (Carrión et al., 2010; Cisler et al., 2015; Geuze et al., 2008; see Table 1). This was present across semantic and episodic memory and during recall of safety cues (extinction retention); however, there is no evidence of similar deficits in other memory/learning systems like acquisition of fear learning, which are largely dependent on other anatomical centers such as amygdala (Janak \& Tye, 2015; Labar et al., 1998).

Interestingly, greater hippocampal activation in PTSD participants as compared to controls has been reported on some memory tasks (Brohawn et al., 2010; Thomaes et al., 2009; Werner et al., 2009). In these studies, greater hippocampal activation was usually associated with memory performance comparable to control groups. One study reported increased hippocampal activation in PTSD compared to controls for negative autobiographical memory and decreased activation for positive autobiographical memory (St. Jacques et al., 2011). Overall, the empirical evidence for "emotional memory" is even more limited, and further research is required to better understand patterns of hippocampal activation during emotional semantic and episodic memory tasks. Whether memory performance/circuits for emotional versus nonemotional items in PTSD differs is still an open question, as it is possible that modulation of the hippocampus by the BLA and $\mathrm{mPFC}$ in emotional memory could contribute to patterns of activation that differ from nonemotional stimuli in PTSD (Pape \& Pare, 2010).

With respect to the role of hippocampus in fear-associated learning in PTSD, the existing human and animal literature clearly suggest that hippocampal-dependent context processing is integral to extinction learning and recall. Decreased activation in PTSD participants has been associated with failure to habituate to $\mathrm{CS}+$ during extinction recall and fear renewal (Garfinkel et al., 2014; Milad et al., 2009). However, as few studies have specifically examined hippocampal function during fear-learning paradigms, further research is needed to establish patterns of deficient hippocampal function during extinction learning, recall, and fear renewal in PTSD.

Evidence from resting state functional connectivity studies in PTSD has been consistent with replicated reports of decreased within-DMN connectivity between the hippocampus and other regions of the DMN, such as the PCC (Miler et al., 2017), and mPFC (Jin et al., 2014; Spielberg et al., 2015). Studies have also demonstrated decreased segregation between DMN and SN regions via the hippocampus (Akiki, Averill, \& Abdallah, 2017; Seeley et al., 2009; Sripada et al., 2012). Deficient connectivity within DMN and less segregation between DMN and SN in PTSD extend findings from task-based analyses and further suggest dysfunction in a network of regions including hippocampus, involved in selfreferential thought, episodic memory, visuospatial imagery, and mental time travel (Greicius et al., 2003).

There are several limitations of this review and the existing literature. First, because we were interested in examining PTSD-associated differences in hippocampal function on a variety of memory and learning tasks, as well as during rest, a meaningful meta-analysis was not feasible. As the literature grows, meta-analyses would be a useful next step to better understand relationships between specific hippocampal function and PTSD symptoms. There have been surprisingly few studies conducted in the past decade focused on hippocampal activation during episodic memory encoding and retrieval, and modulation of fear-associated learning in PTSD. Additional research is needed in these areas. Given that recent reviews have summarized PTSDassociated differences in hippocampal structure, we chose to focus our review on studies using hemodynamic imaging to examine hippocampal function during task and at rest. 


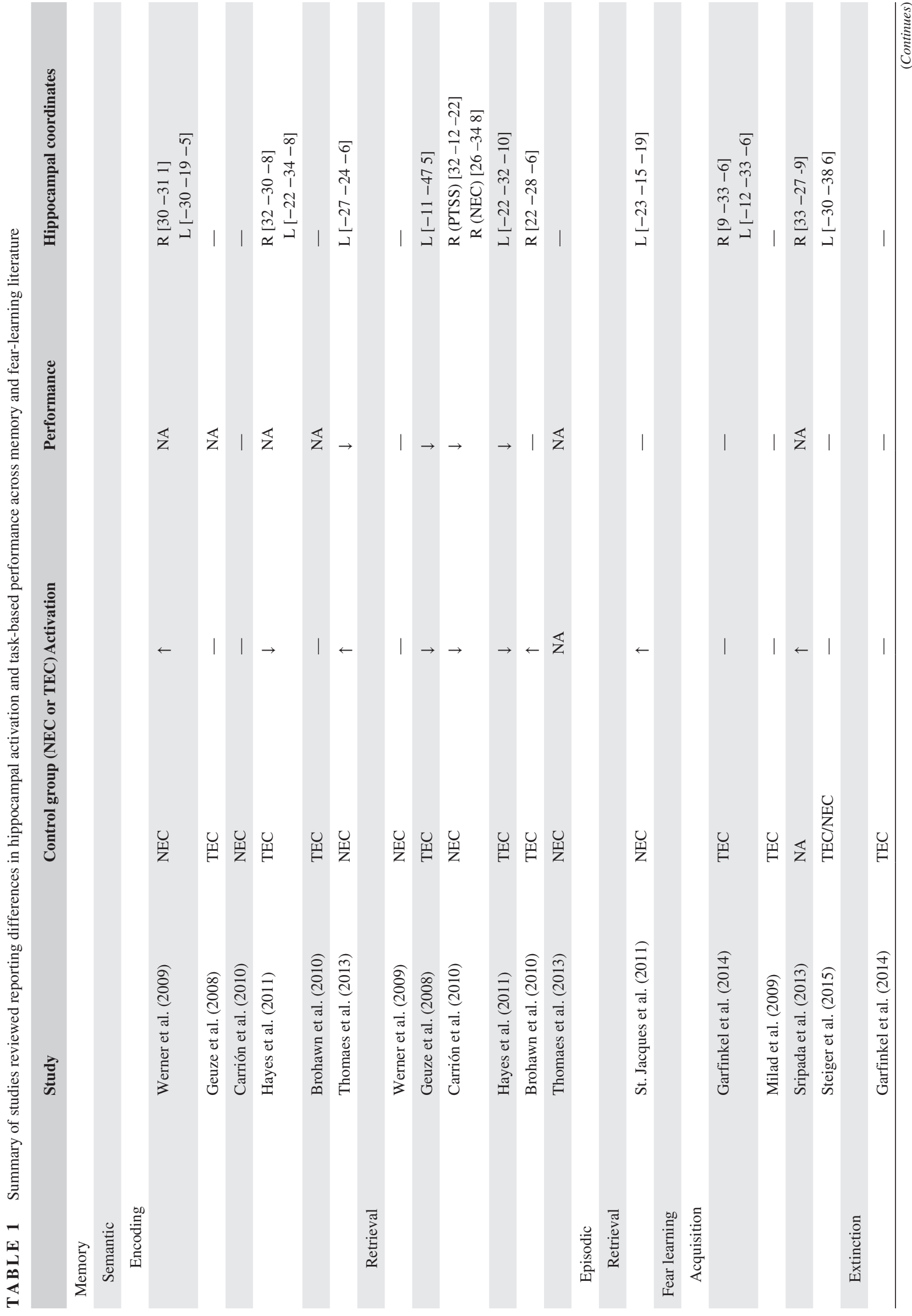


Future studies should continue to examine both hippocampal structure and function associated with performance on memory and learning tasks in PTSD. Studies examining links between hippocampal function and structure would be particularly informative in establishing potential underlying mechanisms associated with performance deficits in PTSD. Recent evidence suggests that contextual processing deficits associated with hippocampal function may be associated with difficulty modulating emotion in participants with PTSD (Liberzon \& Abelson, 2016). Animal studies have already identified differential processes in hippocampal subfields associated with encoding and retrieval of contextual information (Gilbert, Kesner, \& Lee, 2001; Nakashiba et al., 2012). Several examples of human studies, using high resolution neuroimaging techniques, have replicated these findings in healthy adults (Berron et al., 2018; Duncan, Ketz, Inati, \& Davachi, 2012). However, none of the literature reviewed has examined hippocampal subfields or distinguished between activation in anterior and posterior hippocampus. Our own review of the hippocampal coordinates reported in the included studies revealed peaks distributed throughout the hippocampus with few consistencies between task type, likely due to the limited resolution inherent in imaging methods used. Future studies using tasks specifically designed to probe contextual memory associated with hippocampal subfield activation, and high resolution scanning parameters, are needed in both healthy adults and participants with PTSD.

Overall, the evidence emerging from the literature to date suggests that PTSD diagnosis and PTSD symptoms are associated with aberrant (e.g., diminished) hippocampal function during a number of memory and fear-associated learning tasks, as well as deficient connectivity between hippocampus and other regions involved in memory-related processes at rest. Despite recent advances in our understanding of hippocampal involvement in learning and memory processes in PTSD, much remains to be learned. Future efforts should focus on conducting metaanalyses to identify consistent patterns of hippocampal function associated with memory and learning, developing studies to further investigate hippocampal function associated with episodic memory and contextual modulation of fear, examining relationships between hippocampal structure, function, and performance, and utilizing high-resolution imaging techniques to examine hippocampal subfield structure and function in PTSD.

\section{REFERENCES}

Akiki, T. J., Averill, C. L., \& Abdallah, C. G. (2017). A network-based neurobiological model of PTSD: Evidence from structural and functional neuroimaging studies. Current Psychiatry Reports, 19(11), 18. https://doi.org/10.1007/s11920-017-0840-4 
Allen, R. J. (2018). Classic and recent advances in understanding amnesia. F1000Research, 7, 331. https://doi.org/10.12688/ f1000research.13737.1

American Psychiatric Association (2013). Diagnostic and statistical manual of mental disorders (5th ed.). Washington, DC: Author.

Bergstrom, H. C. (2016). The neurocircuitry of remote cued fear memory. Neuroscience and Biobehavioral Reviews, 71, 409-417. https:// doi.org/10.1016/j.neubiorev.2016.09.028

Berron, D., Schütze, H., Maass, A., Cardenas-Blanco, A., Kuijf, H. J., Kumaran, D., \& Düzel, E. (2018). Strong evidence for pattern separation in human dentate gyrus. Journal of Neuroscience, 36(29), 7569-7579. https://doi.org/10.1523/JNEUROSCI.0518-16.2016

Bremner, J. D. (2006). Traumatic stress: Effects on the brain. Dialogues in Clinical Neuroscience, 8(4), 445-461.

Bremner, J. D., Staib, L. H., Kaloupek, D., Southwick, S. M., Soufer, R., \& Charney, D. S. (1999). Neural correlates of exposure to traumatic pictures and sound in Vietnam combat veterans with and without posttraumatic stress disorder: A positron emission tomography study. Biological Psychiatry, 45, 806-816.

Brohawn, K. H., Offringa, R., Pfaff, D. L., Hughes, K. C., \& Shin, L. M. (2010). The neural correlates of emotional memory in posttraumatic stress disorder. Biological Psychiatry, 68(11), 1023-1030. https:// doi.org/10.1016/j.biopsych.2010.07.018

Brown, A., Addis, D. R., Romano, T., Marmar, C., Bryant, R., \& Hirst, W. (2014). Episodic and semantic components of autobiographical memories and imagined future events in post-traumatic stress disorder. Memory, 22, 595-604.

Brown, A. D., Root, J. C., Romano, T. A., Chang, L. J., Bryant, R. A., \& Hirst, W. (2013). Overgeneralized autobiographical memory and future thinking in combat veterans with posttraumatic stress disorder. Journal of Behavior Therapy and Experimental Psychiatry, 44(1), 129-134. https://doi.org/10.1016/j.jbtep.2011.11.004

Carrión, V. G., Haas, B. W., Garrett, A., Song, S., \& Reiss, A. L. (2010). Reduced hippocampal activity in youth with posttraumatic stress symptoms: An fMRI study. Journal of Pediatric Psychology, 35(5), 559-569. https://doi.org/10.1093/jpepsy/jsp112

Cisler, J. M., Bush, K., James, G. A., Smitherman, S., \& Kilts, C. D. (2015). Decoding the traumatic memory among women with PTSD: Implications for neurocircuitry models of PTSD and realtime fMRI neurofeedback. PLOS One, 10(8), e0134717. https://doi. org/10.1371/journal.pone.0134717

Chao, L., Weiner, M., \& Neylan, T. (2013). Regional cerebral volumes in veterans with current versus remitted posttraumatic stress disorder. Psychiatry Research, 213(3), 193-201. https://doi.org/10.1016/j. pscychresns.2013.03.002

Childress, J.E., Mcdowell, E. J., Dalai, V. V., Bogale, S. R., Ramamurthy, C., Jawaid, A., ... Schulz, P. E. (2013). Hippocampal Volumes in patients with chronic combat-related posttraumatic stress disorder: A systematic review. Journal of Neuropsychiatry and Clinical Neurosciences, 25(1), 12-25. https://doi.org/10.1176/appi.neuropsych.12010003

Cohen, N. J., \& Squire, L. R. (1980). Preserved learning and retention of a pattern-analyzing skill in amnesia: Dissociation of knowing how and knowing that. Science, 210, 207-210.

Dere, E., Pause, B. M., \& Pietrowsky, R. (2010). Emotion and episodic memory in neuropsychiatric disorders. Behavioral Brain Research, 215, 162-171. https://doi.org/10.1016/j.bbr.2010.03.017

Duncan, K., Ketz, N., Inati, S. J., \& Davachi, L. (2012). Evidence for area $\mathrm{CA} 1$ as a match/mismatch detector: A high-resolution fMRI study of the human hippocampus. Hippocampus, 22(3), 389-398. https://doi.org/10.1002/hipo.20933

Duval, E., Javanbakht, A., \& Liberzon, I. (2015). Neural circuits in anxiety and stress disorders: A focused review. Therapeutics and Clinical Risk Management, 11, 115-126. https://doi.org/10.2147/ TCRM.S48528

Ehlers, A., \& Clark, D. M. (2000). A cognitive model of posttraumatic stress disorder. Behaviour Research and Therapy, 38, 319-345. https://doi.org/10.1016/S0005-7967(99)00123-0

Eichenbaum, H. (2017). Prefrontal-hippocampal interactions in episodic memory. Nature Reviews Neuroscience, 18(9), 547-558. https://doi.org/10.1038/nrn.2017.74

Eichenbaum, H., \& Cohen, N. J. (2014). Can we reconcile the declarative memory and spatial navigation views on hippocampal function? Neuron, 83(4), 764-770. https://doi.org/10.1016/j. neuron.2014.07.032

Fornito, A., \& Bullmore, E. T. (2010). What can spontaneous fluctuations of the blood oxygenation-level-dependent signal tell us about psychiatric disorders? Current Opinion in Psychiatry, 23(3), 239249. https://doi.org/10.1097/yco.0b013e328337d78

Fox, D., Snyder, A. Z., Barch, D. M., Gusnard, D. A., \& Raichle, M. E. (2005). Transient BOLD responses at block transitions. NeuroImage, 28, 956-966. https://doi.org/10.1016/j.neuroimage.2005.06.025

Frankland, P. W., \& Bontempi, B. (2005). The organization of recent and remote memories. Nature Reviews Neuroscience, 6(2), 119130. https://doi.org/10.1038/nrn1607

Garfinkel, S. N., Abelson, J. L., King, A. P., Sripada, R. K., Wang, X., Gaines, L. M., \& Liberzon, I. (2014). Impaired contextual modulation of memories in PTSD: An fMRI and psychophysiological study of extinction retention and fear renewal. Journal of Neuroscience, 34(40), 13435-13443. https://doi.org/10.1523/jneurosci.4287-13.2014

Geuze, E., Vermetten, E., Ruf, M., de Kloet, C. S., \& Westenberg, H. G. M. (2008). Neural correlates of associative learning and memory in veterans with posttraumatic stress disorder. Journal of Psychiatric Research, 42(8), 659-669. https://doi.org/10.1016/j. jpsychires.2007.06.007

Gilbert, P. E., Kesner, R. P., \& Lee, I. (2001). Dissociating hippocampal subregions: Double dissociation between dentate gyrus and CA1. Hippocampus, 11(6), 626-636. https://doi.org/10.1002/hipo.1077

Gilbertson, M. W., Shenton, M. E., Ciszewski, A., Kasai, K., Lasko, N. B., Orr, S. P., \& Pitman, R. K. (2002). Smaller hippocampal volume predicts pathologic vulnerability to psychological trauma. Nature Neuroscience, 5(11), 1242-1247. https://doi.org/10.1038/nn958. Smaller

Greicius, M. D., Krasnow, B., Reiss, A. L., \& Menon, V. (2003). Functional connectivity in the resting brain: A network analysis of the default mode hypothesis. Proceedings of the National Academy of Sciences of the United States of America, 100(1), 253-258. https://doi.org/10.1073/pnas.0135058100

Hayes, J. P., LaBar, K. S., McCarthy, G., Selgrade, E., Nasser, J., Dolcos, F., ... Morey, R. A. (2011). Reduced hippocampal and amygdala activity predicts memory distortions for trauma reminders in combat-related PTSD. Journal of Psychiatric Research, 45(5), 660-669. https://doi.org/10.1016/j.jpsychires.2010.10.007

Hayes, J. P., Vanelzakker, M. B., \& Shin, L. M. (2012). Emotion and cognition interactions in PTSD: A review of neurocognitive and neuroimaging studies. Frontiers in Integrative Neuroscience, 6(Oct), 89. https://doi.org/10.3389/fnint.2012.00089 
Huijgen, J., \& Samson, S. (2015). The hippocampus: A central node in a large-scale brain network for memory. Revue Neurologique, 171(3), 204-216. https://doi.org/10.1016/j.neurol.2015.01.557

Janak, P. H., \& Tye, K. M. (2015). From circuits to behaviour in the amygdala. Nature, 517(7534), 284-292. https://doi.org/10.1038/ nature14188

Jin, C., Qi, R., Yin, Y., Hu, X., Duan, L., Xu, Q., ... Li, L. (2014). Abnormalities in whole-brain functional connectivity observed in treatment-naive post-traumatic stress disorder patients following an earthquake. Psychological Medicine, 44(9), 1927-1936. https://doi. org/10.1017/S003329171300250X

Jovanovic, T., Kazama, A., Bachevalier, J., \& Davis, M. (2012). Impaired safety signal learning may be a biomarker of PTSD. Neuropharmacology, 62(2), 695-704. https://doi.org/10.1016/j. neuropharm.2011.02.023

Kessler, R. C. (2000). Posttraumatic stress disorder: The burden to the individual and to society. Journal of Clinical Psychiatry, 61(Suppl 5), 4-12.

Kessler, R. C., Berglund, P., Demler, O., Jin, R., Merikangas, K. R., \& Walters, E. E. (2005). Lifetime prevalence and age-of-onset distributions of DSM-IV disorders in the National Comorbidity Survey Replication. Archives of General Psychiatry, 62, 593-602. https:// doi.org/10.1001/archpsyc.62.6.593

Labar, K. S., Gatenby, J., Gore, J. C., Ledoux, J. E., \& Phelps, E. A. (1998). Human amygdala activation during conditioned fear acquisition and extinction: A mixed-trial fMRI study. Neuron, 20(5), 937-945. https://doi.org/10.1016/s0896-6273(00)80475-4

Levy-Gigi, E., Kéri, S., Myers, C. E., Lencovsky, Z., Sharvit-Benbaji, H., Orr, S. P., ... Gluck, M. A. (2012). Individuals with posttraumatic stress disorder show a selective deficit in generalization of associative learning. Neuropsychology, 26(6), 758-767. https://doi. org/10.1037/a0029361

Levy-Gigi, E., Szabo, C., Richter-Levin, G., \& Kéri, S. (2014). Reduced hippocampal volume is associated with overgeneralization of negative context in individuals with PTSD. Neuropsychology, 29(1), 151-161. https://doi.org/10.1037/neu0000131

Liberzon, I., \& Abelson, J. L. (2016). Context processing and the neurobiology of post-traumatic stress disorder. Neuron, 92(1), 14-30. https://doi.org/10.1016/j.neuron.2016.09.039

Logue, M. W., van Rooij, S. J. H., Dennis, E. L., Davis, S. L., Hayes, J. P., Stevens, J. S., .., Morey, R. A. (2018). Smaller hippocampal volume in posttraumatic stress disorder: A multisite ENIGMA-PGC study: Subcortical volumetry results from posttraumatic stress disorder consortia. Biological Psychiatry, 83, 244-253. https://doi. org/10.1016/j.biopsych.2017.09.006

Lopresto, D., Schipper, P., \& Homberg, J. R. (2016). Neural circuits and mechanisms involved in fear generalization: Implications for the pathophysiology and treatment of posttraumatic stress disorder. Neuroscience and Biobehavioral Reviews, 60, 31-42. https://doi. org/10.1016/j.neubiorev.2015.10.009

Maren, S., Phan, K. L., \& Liberzon, I. (2013). The contextual brain: Implications for fear conditioning, extinction and psychopathology. Nature Reviews Neuroscience, 14(6), 417-428. https://doi. org/10.1038/nrn3492

McCormick, C., Rosenthal, C. R., Miller, T. D., \& Maguire, E. A. (2018). Mind-wandering in people with hippocampal damage. Journal of Neuroscience, 38(11), 2745-2754. https://doi.org/10.1523/ jneurosci.1812-17.2018
McEwen, B. S. (1999). Stress and hippocampal plasticity. Annual Review of Neuroscience, 22(1), 105-122. https://doi.org/10.1146/ annurev.neuro.22.1.105

Meyer, T., Quaedflieg, C. W. E. M., Weijland, K., Schruers, K., Merckelbach, H., \& Smeets, T. (2018). Frontal EEG asymmetry during symptom provocation predicts subjective responses to intrusions in survivors with and without PTSD. Psychophysiology, 55(1), 88-108. https://doi.org/10.1111/psyp.12779

Milad, M. R., Pitman, R. K., Ellis, C. B., Gold, A. L., Shin, L. M., Lasko, N. B., ... Rauch, S. L. (2009). Neurobiological basis of failure to recall extinction memory in posttraumatic stress disorder. Biological Psychiatry, 66(12), 1075-1082. https://doi.org/10.1016/j. biopsych.2009.06.026

Milad, M. R., Wright, C. I., Orr, S. P., Pitman, R. K., Quirk, G. J., \& Rauch, S. L. (2007). Recall of fear extinction in humans activates the ventromedial prefrontal cortex and hippocampus in concert. Biological Psychiatry, 62(5), 446-454. https://doi.org/10.1016/j. biopsych.2006.10.011

Miler, D. R., Hayes, S. M., Hayes, J. P., Spielberg, J. M., Lafleche, G., \& Verfaellie, M. (2017). Default mode network subsystems are differentially disrupted in posttraumatic stress disorder. Biological Psychiatry: Cognitive Neuroscience and Neuroimaging, 2(4), 363371. https://doi.org/10.1016/j.bpsc.2016.12.006

Mitra, A., \& Raichle, M. E. (2016). How networks communicate: Propagation patterns in spontaneous brain activity. Philosophical Transactions of the Royal Society B: Biological Sciences, 371(1705), 20150546. https://doi.org/10.1098/rstb.2015.0546

Nadel, L., \& Moscovitch, M. (1997). Memory consolidation, retrograde amnesia and the hippocampal complex. Current Opinions in Neurobiology, 7, 217-227. https://doi.org/10.1016/ S0959-4388(97)80010-4

Nakashiba, T., Cushman, J. D., Pelkey, K. A., Renaudineau, S., Buhl, D. L., McHugh, T. J., ... Tonegawa, S. (2012). Young dentate granule cells mediate pattern separation, whereas old granule cells facilitate pattern completion. Cell, 149(1), 188-201. https://doi.org/10.1016/j. cell.2012.01.046

Nelson, M., \& Tumpap, A. (2017). Posttraumatic stress disorder symptom severity is associated with left hippocampal volume reduction: A meta-analytic study. CNS Spectrums, 22(4), 363-372. https://doi. org/10.1017/S1092852916000833

Niibori, Y., Yu, T.-S., Epp, J. R., Akers, K. G., Josselyn, S. A., \& Frankland, P. W. (2012). Suppression of adult neurogenesis impairs population coding of similar contexts in hippocampal CA3 region. Nature Communications, 3, 1253. https://doi.org/10.1038/ncomms2261

Opitz, B. (2014). Memory function and the hippocampus. Frontiers of Neurology and Neuroscience. The Hippocampus in Clinical Neuroscience, 34, 51-59. https://doi.org/10.1159/000356422

Pape, H.-C., \& Pare, D. (2010). Plastic synaptic networks of the amygdala for the acquisition, expression, and extinction of conditioned fear. Physiology Review, 90, 419-463. https://doi.org/10.1152/ physrev.00037.2009

Patel, R., Spreng, R. N., Shin, L. M., \& Girard, T. A. (2012). Neurocircuitry models of posttraumatic stress disorder and beyond: A meta-analysis of functional neuroimaging studies. Neuroscience and Biobehavioral Reviews, 36, 2130-2142. https:// doi.org/10.1016/j.neubiorev.2012.06.003

Patriat, R., Birn, R. M., Keding, T. J., \& Herringa, R. J. (2016). Defaultmode network abnormalities in pediatric posttraumatic stress disorder. 
Journal of the American Academy of Child and Adolescent Psychiatry, 55(4), 319-327. https://doi.org/10.1016/j.jaac.2016.01.010

Power, J. D, Cohen, A. L, Nelson, S. M, Wig, G. S, Barnes, K. A., Church, J. A., ... Petersen, S. E. (2011). Functional network organization of the human brain. Neuron, 72(4), 665-678.

Rabinak, C. A., MacNamara, A., Kennedy, A. E., Angstadt, M., Stein, M. B., Liberzon, I., \& Phan, L. (2014). Focal and aberrant prefrontal engagement during emotion regulation in veterans with posttraumatic stress disorder. Depression and Anxiety, 31, 851-861.

Samuelson, K. W. (2011). Post-traumatic stress disorder and declarative memory functioning: A review. Dialogues in Clinical Neuroscience, 13(3), 346-351.

Sapolsky, R. M. (2000). Glucocorticoids and hippocampal atrophy in neuropsychiatric disorders. Archives of General Psychiatry, 57(10), 925-935. https://doi.org/10.1001/archpsyc.57.10.925

Scoville, W. B., \& Milner, B. (1957). Loss of recent memory after bilateral hippocampal lesions. Journal of Neurology, Neurosurgery \& Psychiatry, 20(1), 11-21. https://doi.org/10.1136/jnnp.20.1.11

Seeley, W. W., Menon, V., Schatzberg, A. F., Keller, J., Gary, H., Kenna, H., \& Greicius, M. D. (2009). Dissociable intrinsic connectivity networks for salience processing and executive control. NIH Public Access, 27(9), 2349-2356. https://doi.org/10.1523/ JNEUROSCI.5587-06.2007

Sharot, T., \& Yonelinas, A. P. (2008). Differential time-dependent effects of emotion on recollective experience and memory for contextual information. Cognition, 106(1), 538-547. https://doi.org/10.1016/j. cognition.2007.03.002

Sierra-Mercado, D., Padilla-Coreano, N., \& Quirk, G. J. (2011). Dissociable roles of prelimbic and infralimbic cortices, ventral hippocampus, and basolateral amygdala in the expression and extinction of conditioned fear. Neuropsychopharmacology, 36(2), 529-538. https://doi.org/10.1038/npp.2010.184

Shin, L. M., \& Liberzon, I. (2010). The neurocircuitry of fear, stress, and anxiety disorders. Neuropsychopharmacology, 35(1), 169-191. https://doi.org/10.1038/npp.2009.83

Spielberg, J. M., McGlinchey, R. E., Milberg, W. P., \& Salat, D. H. (2015). Brain network disturbance related to posttraumatic stress and traumatic brain injury in veterans. Biological Psychiatry, 78(3), 210-216. https://doi.org/10.1016/j.biopsych.2015.02.013

Sripada, R. K., Garfinkel, S. N., \& Liberzon, I. (2013). Avoidant symptoms in PTSD predict fear circuit activation during multimodal fear extinction. Frontiers in Human Neuroscience, 7, https://doi. org/10.3389/fnhum.2013.00672

Sripada, R. K., King, A. P., Welsh, R. C., Garfinkel, S. N., Wang, X., Sripada, C. S., \& Liberzon, I. (2012). Neural dysregulation in posttraumatic stress disorder: Evidence for disrupted equilibrium between salience and default mode brain networks. Psychosomatic Medicine, 74(9), 904-911. https://doi.org/10.1097/PSY.0b013e318273bf33

St. Jacques, P. L., Botzung, A., Miles, A., \& Rubin, D. C. (2011). Functional neuroimaging of emotionally intense autobiographical memories in post-traumatic stress disorder. Journal of Psychiatric Research, 45(5), 630-637. https://doi.org/10.1016/j.jpsychires.2010.10.011

Steiger, F., Nees, F., Wicking, M., Lang, S., \& Flor, H. (2015). Behavioral and central correlates of contextual fear learning and contextual modulation of cued fear in posttraumatic stress disorder. International Journal of Psychophysiology, 98(3), 584-593. https:// doi.org/10.1016/j.ijpsycho.2015.06.009

Tamburrino, M. B., Chan, P., Prescott, M., Calabrese, J., Liberzon, I., Slembarski, R., ... Galea, S. (2015). Baseline prevalence of Axis I diagnosis in the Ohio Army National Guard. Psychiatry Research, 226(1), 142-148. https://doi.org/10.1016/j.psychres.2014.12.038

Thomaes, K., Dorrepaal, E., Draijer, N. P. J., DeRuiter, M. B., Elzinga, B. M., VanBalkom, A. J., ... Veltman, D. J. (2009). Increased activation of the left hippocampus region in complex PTSD during encoding and recognition of emotional words: A pilot study. Psychiatry Research, 171(1), 44-53.

Thomaes, K., Dorrepaal, E., Draijer, N., deRuiter, M. B., Elzinga, B. M., Sjoerds, Z., ... Veltman, D. J. (2013). Increased anterior cingulate cortex and hippocampus activation in complex PTSD during encoding of negative words. Social Cognitive and Affective Neuroscience, 8(2), 190-200. https://doi.org/10.1093/scan/nsr084

Thompson, W. W., Gottesman, I. I., \& Zalewski, C. (2006). Reconciling disparate prevalence rates of PTSD in large samples of US male Vietnam veterans and their controls. BMC Psychiatry, 6, 19. https:// doi.org/10.1186/1471-244X-6-19

Vaidya, C. J., \& Gordon, E. M. (2013). Phenotypic variability in restingstate functional connectivity: Current status. Brain Connectivity, 3(2), 99-120. https://doi.org/10.1089/brain.2012.0110

Van Rooij, S. J. H., Kennis, M., Sjouwerman, R., van den Heuvel, M. P., Kahn, R. S., \& Geuze, E. (2015). Smaller hippocampal volume as a vulnerability factor for the persistence of post-traumatic stress disorder. Psychological Medicine, 45(13), 2737-2746. https://doi. org/10.1017/S0033291715000707

Vasterling, J. J., Duke, L. M., Brailey, K., Constans, J. I., Allain, A. N., \& Sutker, P. B. (2002). Attention, learning, and memory performances and intellectual resources in Vietnam veterans: PTSD and no disorder comparisons. Neuropsychology, 16(1), 5-14. https://doi. org/10.1037//0894-4105.16.1.5

Werner, N. S., Meindl, T., Engel, R. R., Rosner, R., Riedel, M., Reiser, M., \& Fast, K. (2009). Hippocampal function during associative learning in patients with posttraumatic stress disorder. Journal of Psychiatric Research, 43(3), 309-318. https://doi.org/10.1016/j. jpsychires.2008.03.011

Wicking, M., Steiger, F., Nees, F., Diener, S. J., Grimm, O., Ruttorf, M., ... Flor, H. (2016). Deficient fear extinction memory in posttraumatic stress disorder. Neurobiology of Learning and Memory, 136, 116-126. https://doi.org/10.1016/j.nlm.2016.09.016

Wotjak, C. T., \& Pape, H.-C. (2013). Neuronal circuits of fear memory and fear extinction. E-Neuroforum, 19(3), 47-56. https://doi. org/10.1007/s13295-013-0046-0

How to cite this article: Joshi SA, Duval ER, Kubat B, Liberzon I. A review of hippocampal activation in post-traumatic stress disorder. Psychophysiology. 2020;57:e13357. https://doi.org/10.1111/psyp.13357 